\title{
BOUNDARY BEHAVIOR OF STARLIKE FUNCTIONS
}

\section{P. J. EENIGENBURG}

AbSTRACT. For a starlike function $f$, we impose a geometric condition on the image of the open unit disc by the mapping $w=z f^{\prime}(z) / f(z)$ to insure that $f$ be one-to-one on the closed unit disc. Applications are given to certain classes of univalent functions, including spiral-like functions.

1. Introduction. A function

$$
f(z)=\sum_{n=1}^{\infty} a_{n} z^{n}
$$

analytic in $|z|<1$ is said to be starlike there if it maps $|z|<1$ one-to-one onto a domain starshaped with respect to the origin. This is equivalent to the analytic condition that $\operatorname{Re}\left[z f^{\prime}(z) \mid f(z)\right]>0$ in $|z|<1$. Moreover, if there exists $\alpha, 0<\alpha<1$, such that $\operatorname{Re}\left[z f^{\prime}(z) \mid f(z)\right]>\alpha$ for $|z|<1$, we say that $f$ is starlike of order $\alpha$.

An analytic function (1) is said to be convex if it maps $|z|<1$ one-to-one onto a convex domain. Analytically, this is equivalent to the condition that $\operatorname{Re}\left[1+z f^{\prime \prime}(z) / f^{\prime}(z)\right]>0$ for $|z|<1$.

Henceforth, let $D$ denote the open unit disc. It is clear that a bounded convex function maps $D$ onto a domain whose boundary, $\partial f(D)$, is a rectifiable closed curve. However, if $f$ is a bounded starlike function, then $\partial f(D)$ need not be rectifiable. For example, let $\left\{\theta_{n}\right\}_{n=1}^{\infty}$ be a sequence of values of $\theta$ such that $0 \leqq \theta_{1}<\theta_{2}<\cdots<\theta_{n}<\cdots<\pi$, and $\lim _{n \rightarrow \infty} \theta_{n}=\pi$. Let $l_{n}$ be the linear segment $\left[\frac{1}{2} e^{i \theta_{n}}, e^{i \theta_{n}}\right)$, and $l_{0}=\left[\frac{1}{2} e^{i \pi}, e^{i \pi}\right)$. Then, if $E=$ $D \bigcup_{n=0}^{\infty} l_{n}$, the Riemann mapping theorem guarantees the existence of a univalent function $f_{1}$ such that $f_{1}(D)=E$ and $f_{1}(0)=0$. Since $E$ is a domain which is starlike with respect to the origin, it follows that $f_{1}$ is a starlike function. Furthermore, $\partial E$ is not rectifiable.

It is known [7] that if $f$ is convex then $f$ is starlike of order $\frac{1}{2}$. Now the following question arises. If $f$ is starlike of some positive order, does the boundedness of $f$ imply that $\partial f(D)$ is rectifiable? Since

$$
\operatorname{Re}\left(\frac{z f^{\prime}(z)}{f(z)}\right)=\frac{\partial}{\partial \theta} \arg f\left(r e^{i \theta}\right), \quad 0<r<1,
$$

Received by the editors October 5, 1970 and, in revised form, June 16, 1971. AMS 1970 subject classifications. Primary 30A32; Secondary 30A72. Key words and phrases. Univalent function, starlike, convex. 
the fact that $f$ is starlike of order $\alpha>0$ implies that

$$
\frac{\partial}{\partial \theta} \arg f\left(r e^{i \theta}\right)>\alpha, \quad 0<r<1
$$

Hence, one perhaps has reason to believe that $\arg f\left(r e^{i \theta}\right)$ increases rapidly enough, as a function of $\theta$, so that a rectifiable boundary must result. However, this need not be the case. Simply let $\alpha$ satisfy $0<\alpha<1$ and define $g_{1}$ by

$$
g_{1}(z)=z\left(f_{1}(z) / z\right)^{1-\alpha} \text {. }
$$

Then $g_{1}$ is starlike of order $\alpha$, but $\partial g_{1}(D)$ is not rectifiable. In fact, $g_{1}$ does not extend continuously to $\bar{D}$.

2. Boundary behavior. As noted above, there exist bounded starlike functions of any given order $\alpha, 0<x<1$, which are not continuous on $\bar{D}$. If $g$ is such a function, a theorem of Carathéodory states that $\partial g(D)$ is not a Jordan curve. But $\partial g(D)$ fails to be a Jordan curve in a nontrivial fashion; for if $g$ is starlike of order $\alpha, \alpha>0$, then the finite radia! limits of $g$ are distinct. This result is contained in the following theorem.

THEOREM 1. Let $f$ be starlike in $D$, and let $\hat{f}(\theta)=\lim _{r \rightarrow 1} f\left(r e^{i \theta}\right) .^{1}$ If the intersection of the imaginary axis, $I$, with the closure of the set $\left\{w=z f^{\prime}(z) / f(z): z \in D\right\}$ is not a neighborhood (in I) of the origin, then $\hat{f}$ is one-to-one on the set of $\theta$ in $[-\pi, \pi)$ for which $\hat{f}(\theta)$ is finite.

Proof. By the familiar Herglotz representation, there exists a nondecreasing function $V(\theta)$ such that $V(\pi)-V(-\pi)=2 \pi$ and

$$
\frac{z f^{\prime}(z)}{f(z)}=\frac{1}{2 \pi} \int_{-\pi}^{\pi} \frac{1+z e^{-i t}}{1-z e^{-i t}} d V(t) .
$$

Assuming $f^{\prime}(0)>0$ (without loss of generality), Keogh [2] has shown that it is possible to normalize $V(t)$ so that, for a certain fixed branch of $\arg f(z)$ and for all real $\theta$,

$$
V(\theta)=\lim _{r \rightarrow 1} \arg f\left(r e^{i \theta}\right)^{2}
$$

Suppose there exist $\theta_{1}, \theta_{2},-\pi \leqq \theta_{1}<\theta_{2}<\pi$, such that $\hat{f}\left(\theta_{1}\right)=\hat{f}\left(\theta_{2}\right) \neq \infty$. Since $V(\theta)$ is nondecreasing, $\bar{V}(\theta)$ is constant on either $\left[\theta_{1}, \theta_{2}\right]$ or $\left[\theta_{2}, \theta_{1}+2 \pi\right]$. Assuming the former, it follows from (2) that $z f^{\prime}(z) / f(z)$ is

\footnotetext{
1 By a theorem of Keogh [2], $\hat{f}(\theta)$ exists (possibly infinite) for all real values of $\theta$.

${ }^{2}$ We define $V(\theta)$ for all $\theta$ by the condition $V(\theta+2 \pi)-V(\theta)=2 \pi$.
} 
analytic on the arc $\left(e^{i \theta_{1}}, e^{i \theta_{2}}\right)$ and has zero real part there. Again from (2),

$$
\log \left(\frac{f(z)}{f^{\prime}(0) z}\right)=\frac{1}{2 \pi} \int_{-\pi}^{\pi} \log \frac{1}{\left(1-z e^{-i t}\right)^{2}} d V(t) .
$$

Thus, $f$ is analytic on $\left(e^{i \theta_{1}}, e^{i \theta_{2}}\right)$ and, in view of (3), the values taken by $f$ on this arc fill a segment of some ray emanating from the origin. Taking real parts in (4), it follows by Rolle's theorem that there exists $\theta_{0} \in\left(\theta_{1}, \theta_{2}\right)$ such that $(\partial / \partial \theta) \log |\hat{f}(\theta)|=0$ at $\theta=\theta_{0}$. Hence, $\operatorname{Im}\left(z f^{\prime}(z) / f(z)\right)=0$ at $z=e^{i \theta_{0}}$; and so, there exists an open interval of the imaginary axis which contains the origin and belongs to the closure of $\left\{w=z f^{\prime}(z) / f(z): z \in D\right\}$. The proof is complete.

One may ask whether the converse is true. That is, if the intersection of the imaginary axis, $I$, with the closure of $\left\{w=z f^{\prime}(z) / f(z): z \in D\right\}$ is a neighborhood (in $I$ ) of the origin, does $\hat{f}$ fail to be one-to-one on the set of $\theta$ in $[-\pi, \pi)$ for which $\hat{f}(\theta)$ is finite? The answer is no, in general. In fact, let $G$ be any simply connected domain (containing the point 1) lying in the right half plane, having a prime end that is a neighborhood (in I) of the origin; and let $g$ be a Riemann mapping function from $D$ onto $G$, $g(0)=1$. If $f$ is any solution of the differential equation $z f^{\prime}(z)=g(z) f(z),{ }^{3}$ $f$ is starlike in $D$ and $\hat{f}$ is one-to-one on the set of $\theta$ in $[-\pi, \pi)$ for which $\hat{f}(\theta)$ is finite. This follows by the same argument used in Theorem 1 .

Theorem 1 and a well-known theorem of Carathéodory yield the following corollary.

COROLlaRY. Under the hypotheses of Theorem 1, the following are equivalent:

(a) $f$ is continuous on $\tilde{D}$.

(b) $\partial f(D)$ is a Jordan curve.

3. Examples. The following classes of functions satisfy the conditions of Theorem 1.

A. Starlike functions of order $\alpha, 0<\alpha<1$.

B. Strongly starlike functions of order $\alpha, 0<\alpha<1$.

By definition, the starlike function $f$ is said to be strongly starlike of order $\alpha(0<\alpha<1)$ if

$$
\left|\arg \left(z f^{\prime}(z) / f(z)\right)\right| \leqq \alpha(\pi / 2), \quad z \in D .
$$

This class of functions has recently been examined by Brannan and Kirwan [1]. They show that (a) and (b) of the corollary hold for this class. In fact, if $f$ is strongly starlike of order $\alpha(0<\alpha<1)$, then $f(D)$ has a rectifiable boundary curve.

\footnotetext{
${ }^{3}$ The function $z \exp \int_{0}^{z}(g(n)-1) w^{-1} d w$ is easily seen to be a solution.
} 
C. We now consider a class of functions which satisfies a "rotated" form of Theorem 1.

Definition. If $f$ be analytic in $D, f^{\prime}(0) \neq 0$, then $f$ is said to be $\alpha$ spiral-like, $-\pi / 2<\alpha<\pi / 2$, if

$$
\operatorname{Re}\left(e^{i \alpha} z f^{\prime}(z) / f(z)\right)>0, \quad z \in D .
$$

If $\alpha=0$ we have the class of starlike functions. Spaček [6] has shown that if an analytic function $f$ satisfies (5), then $f$ must be univalent in $D$. Further properties of $x$-spiral-like functions can be found in [3]. Note that (5) is equivalent to the statement that the image of $D$ under the mapping $z f^{\prime}(z) / f(z)$ should lic to the right of the line $w=t e^{i(\pi / 2-\alpha)}, t$ real.

If $f$ is $\alpha$-spiral-like, the function $g$, defined by

$$
g(z)=z(f(z) / z)^{1+i \tan \alpha 4}, \quad z \in D,
$$

is starlike in $D$. It follows that $f$ has radial limits (possibly infinite) for all values of $\theta$. Furthermore, Theorem 1 and the corollary hold for $\alpha$-spirallike functions, if one replaces " $I$ " by "line $w=t e^{i(\pi / 2-\alpha)}, t$ real."

The following theorem illustrates the geometric distinction between spiral-like and starlike functions.

THEOREM 2. Suppose $f$ is both $\alpha$ and $\beta$-spiral-like, $-\pi / 2<\alpha<\beta<\pi / 2$. Then $f$ is $\gamma$-spiral-like, $\forall \gamma, \alpha \leqq \gamma \leqq \beta$; and $f$ maps $D$ onto a domain whose buundary is a rectifiable Jordan curve.

Proof. The fact that $\operatorname{Re}\left(e^{i \gamma} z f^{\prime}(z) / f(z)\right)>0$ for $z \in D$ follows from the geometry of the situation. Furthermore, there exists $\rho, 0<\rho<1$, such that

$$
\frac{z f^{\prime}(z)}{f(z)}<\left[1-z e^{i(\pi-(\alpha+\beta)) / 2}\right]^{\rho}\left[1-z e^{-i(\pi-(\alpha+\beta)) / 2}\right]^{-\rho} .
$$

By an inequality of Littlewood [4], we have

$$
\int_{-\pi}^{\pi}\left|\frac{z f^{\prime}(z)}{f(z)}\right| d \theta \leqq 2^{\rho} \int_{-\pi}^{\pi}\left|1-r e^{i \theta}\right|^{-\rho} d \theta .
$$

Since $0<\rho<1, \int_{-\pi}^{\pi}\left|z f^{\prime}(z)\right| f(z) \mid d \theta$ remains bounded as $r \rightarrow 1$, and so $z f^{\prime}(z) / f(z) \in H$. It follows also from (6) that $f$ is bounded; thus $f^{\prime} \in H$. By a theorem of Seidel [5], $\partial f(D)$ is rectifiable. Also, (6) shows that the hypotheses of the spiral-like formulation of Theorem 1 hold; hence, part (a) of the corollary holds (since $f^{\prime} \in H$ ). The proof is complete.

The author is indebted to the referee for helpful comments regarding Theorem 1.

All powers are meant as principal values. 


\section{REFERENCES}

1. D. A. Brannan and W. E. Kirwan, On some classes of bounded univalent functions, J. London Math. Soc. (2) 1 (1969), 431-443. MR 40 \#4439.

2. F. R. Keogh, Some theorems on conformal mapping of bounded star-shaped domains, Proc. London Math. Soc. (3) 9 (1959), 481-491. MR 22 \#1683.

3. R. J. Libera, Univalent $\alpha$-spiral functions, Canad. J. Math. 19 (1967), 449-456. MR 35 \#5599.

4. J. E. Littlewood, On inequalities in the theory of functions, Proc. London Math. Soc. 23 (1925), 481-519.

5. W. Seidel, Über die Ränderzuordnung bei konformen Abbildungen, Math. Ann. 104 (1931), 182-243.

6. L. Špaček, Contribution à la théorie des fonctions univalentes, Casopis Pěst. Mat. 62 (1933), 12-19.

7. E. Strohhäcker, Beitrage zur Theorie des schlichten Funktionen, Math. Z. 37 (1933), 356-380.

Department of Mathematics, Western Michigan University, Kalamazoo, MICHIGAN 49001 